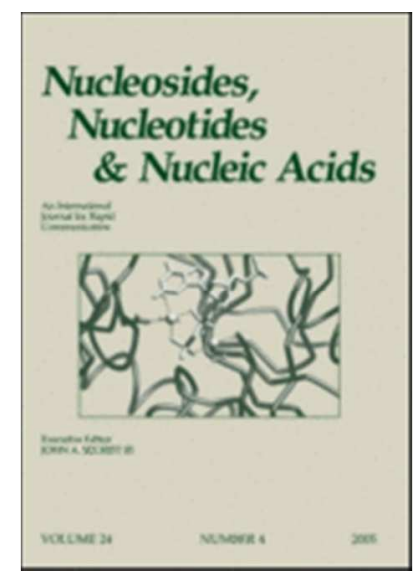

\title{
The development of an in vitro cerebral organoid model for investigating the pathomolecular mechanisms associated with the Central Nervous System involvement in Mitochondrial Neurogastrointestinal Encephalomyopathy
}

\begin{tabular}{|r|l|}
\hline Journal: & Nucleosides, Nucleotides and Nucleic Acids \\
\hline Manuscript ID & LNCN-2018-0073 \\
\hline Manuscript Type: & Conference Proceedings \\
\hline Date Submitted by the Author: & 05-Jun-2018 \\
\hline Complete List of Authors: & $\begin{array}{l}\text { Pacitti, Dario; St George's, University of London, Molecular and Clinical } \\
\text { Sciences Research Institute } \\
\text { Bax, Bridget E.; St George's, University of London, Molecular and Clinical } \\
\text { Sciences Research Insitute }\end{array}$ \\
\hline Keywords: & $\begin{array}{l}\text { Cerebral organoids, Mitochondrial Neurogastrointestinal } \\
\text { Encephalomyopathy, Thymidine phosphorylase, Disease modeling, Induced } \\
\text { pluripotent stem cells }\end{array}$ \\
\hline &
\end{tabular}

\section{SCHOLARONE \\ Manuscripts}




\begin{abstract}
Declaration
I, Dario Pacitti, hereby declare that the following manuscript has not been previously published nor submitted simultaneously for publication to any other journal.
\end{abstract}




\begin{abstract}
Title
The development of an in vitro cerebral organoid model for investigating the pathomolecular mechanisms associated with the Central Nervous System involvement in Mitochondrial Neurogastrointestinal Encephalomyopathy (MNGIE)
\end{abstract}

\title{
Short title
}

Development of a Cerebral organoid model for MNGIE

Authors

Pacitti D., Bax B.E 


\section{Abstract}

Mitochondrial neurogastrointestinal encephalomyopathy (MNGIE) is a rare disorder caused by mutations in the thymidine phosphorylase gene (TYMP), leading to secondary aberrations to the mitochondrial genome. The disease is characterised by gastrointestinal dysmotility, sensorimotor peripheral neuropathy and leukoencephalopathy. The understanding of the molecular mechanisms that underlie the central nervous system (CNS) is hindered by the lack of a representative disease model; to address this we have developed an in vitro 3-D cerebral organoid of MNGIE.

Induced pluripotent stem cells (iPSCs) generated from peripheral blood mononuclear cells (PBMC) of a healthy control and a patient with MNGIE were characterised to ascertain bona fide pluripotency through the evaluation of pluripotency markers and the differentiation to the germ layers. iPSC lines were differentiated into cerebral organoids. Thymidine phosphorylase expression in PBMCs, iPSCs and Day 92 organoids was evaluated by immunoblotting and intact organoids were sampled for histological evaluation of neural markers.

iPSCs demonstrated the expression of pluripotency markers SOX2 and TRA1-60 and the plasticity to differentiate into the germ layers. Cerebral organoids stained positive for the neural markers GFAP, O4, Tuj1, Nestin, SOX2 and MBP. Consistent with the disease phenotypes, MNGIE cells did not display thymidine phosphorylase expression whereas control PBMCs and Day 92 organoids did. Remarkably, control iPSCs did not stain positive for thymidine phosphorylase.

We have established for the first time a MNGIE iPSC line and cerebral organoid model, which exhibited the expression of cells relevant to the study of the disease, such as neural stem cells, astrocytes and myelinating oligodendrocytes. 


\section{Introduction}

Mitochondrial neurogastrointestinal encephalomyopathy (MNGIE) is an ultra- rare inherited disease which manifests clinically as severe gastrointestinal dysmotility, sensorimotor peripheral neuropathy, severe muscle weakness, and progressive leukoencephalopathy as observed by Magnetic Resonance Imaging (MRI). MNGIE is relentlessly progressive, with patients usually dying from a combination of nutritional failure and muscular disability at an average age of 37.6 years [1]. The disorder is caused by mutations in the nuclear gene TYMP, which results in a deficiency in the enzyme thymidine phosphorylase and an accumulation in the deoxyribonucleosides, thymidine and deoxyuridine in cellular and extracellular compartments [2]. This ultimately leads to elevated intracellular concentrations of their corresponding triphosphates, creating imbalances in the mitochondrial deoxyribonucleoside triphosphate pools, and results in multiple deletions, somatic point mutations and depletion of mitochondrial DNA (mtDNA) [3-6].

The synthesis of mtDNA in post-mitotic cells is dependent on the supply of deoxyribonucleoside triphosphates via the mitochondrial nucleotide salvage pathway and thus tissues such as the skeletal muscle and nervous system are most affected in MNGIE.

There is paucity of information on the underlying molecular perturbations of MNGIE and how these influence the disease phenotype; the rarity of the disease and its high mortality rate impose constraints on the availability of biological samples for analysis. The absence of a disease-specific model that accurately reflects the clinical features of MNGIE has contributed to the lack of advancement of knowledge into the disorder. Indeed, in vitro models employed to date have exploited the use of cells that are not relevant to the systems affected in MNGIE, with a majority of the studies employing cells from healthy individuals. Although these studies have provided some understanding of how the nucleotide pool imbalances contribute to mtDNA derangements, none have so far developed relevant cell lines of the nervous and enteric systems with MNGIE genotypes [7-9]. The study of how the absence of thymidine phosphorylase expression perturbs other biological 
pathways involved in nervous tissue development and maintenance, has therefore been neglected. Murine models based on the double knock-out of $T y m p^{-/} / \mathrm{Upp}^{-/}$genes have been used to recapitulate some features of MNGIE, however these also displayed incongruences with the clinical scenario [10-12]. Differences in the deoxyribonucleoside metabolism between humans and mice further support the inadequacy of this model in recapitulating the human disease [10-12].

Patient-derived induced pluripotent stem cells (iPSCs) provide access to cell types that were previously unobtainable in sufficient quantity or quality and present the opportunity to elucidate the underlying molecular mechanisms that contribute to the tissue-specific aspects of MNGIE disease. In addition to offering cells with the same genotype of the donor, iPSCs possess the plasticity to differentiate into virtually any tissue type [13].

In recent years, advances in culturing techniques has enabled the use of iPSCs for the generation of 3-dimensional culture systems consisting of different interacting cell types, reminiscent of tissue-like structures. These culture platforms, defined as organoids, allow the in vitro analysis of molecular, cellular and biological phenotypes as well as the study of organogenesis; examples of organoids generated from iPSCs include those representing the intestine, lung, liver, kidney, ovary and cerebrum [14].

One of the questions that remain unanswered is the relevance of white matter lesions in MNGIE. In this study we developed cerebral organoids, characterized by the presence of diversified cell types including glial and neuronal cells and hypothesised they would provide a mechanistic model for investigating the central nervous system (CNS) involvement in MNGIE. 


\section{Methods \\ iPSC generation and maintenance}

All reagents were purchased from Life Technologies, UK, unless mentioned otherwise. Whole blood was collected from a healthy individual and a patient with MNGIE, who was previously reported by Filosto et al. [15] and Bax et al. [16], from which peripheral blood mononuclear cells (PBMCs) were isolated using the BD Cell Preparation Tubes (BD, UK) as indicated by the manufacturer. PBMC fractions were reprogrammed using non-integrating, non-viral vectors for the delivery of the Yamanaka factors as described by Okita et al. [17]. To generate healthy control iPSCs, $4 \mu \mathrm{g}$ of pCXLEhOCT3/4-p53shRNA, pCXLE-hUL, and pCXLE-hSK and $2 \mu \mathrm{g}$ pCXB-EBNA1 (Addgene, UK) were electroporated into $2 \times 10^{6}$ PBMCs using a BTX ECM600, set to deliver 1 pulse of $300 \mathrm{~V}$ for $20 \mathrm{mS}$. For the generation of MNGIE iPSCs, an optimised protocol was used, where $3.32 \mu \mathrm{g}$ of pCXLE-hOCT3/4p53shRNA, pCXLE-hUL, and pCXLE-hSK and $0.5 \mu \mathrm{g}$ pCXB-EBNA1 were electroporated into $1 \times 10^{5}$ PBMCs using the Neon system. Conditions used were $2150 \mathrm{~V}, 20 \mathrm{mS}$ and 1 pulse. Transfected PBMCs were then plated onto mouse embryonic fibroblasts (MEFs) coated $35 \mathrm{~mm}$ dishes in Opti-MEM supplemented with $20 \%$ Knock-out Serum replacement (KOSR), 1:100 non-essential amino acids (NEAA) and $10 \mu \mathrm{M}$ of $\mathrm{Y}-27632$ (Tocris Bioscience, UK). Forty-eight hours post-transfection an equal volume of iPSC medium, consisting of DMEM: Nutrient Mixture F12 supplemented with 20\% KOSR, 1:100 NEAA, 1\% Penicillin/Streptomycin, 0.2\% Plasmocin (Invivogen, USA), $0.14 \quad m M \quad \beta-$ mercaptoethanol (Sigma-Aldrich, UK), $10 \mathrm{ng} / \mathrm{mL}$ b-FGF2 (Peprotech, UK) and $10 \mu \mathrm{M}$ Y-27632. After an additional 48 hours the spent media was changed to $4 \mathrm{~mL}$ of fresh iPSC medium without Y-27632 and replaced daily. iPSCs were cultured up to $80 \%$ confluence and sub-cultured mechanically under a light microscope in a biosafety hood. For differentiation assays, iPSCs were transitioned to xeno-free cultures by dissociating cells into single cell suspensions with Accutase (Sigma-Aldrich, UK) and Y27632. Single cell suspensions were then plated onto $35 \mathrm{~mm}$ dishes coated with $5 \mu \mathrm{g} / \mathrm{mL}$ Vitronectin and cultured in E8 medium. Sub-culturing of xeno-free iPSCs was performed by incubation in Versene for 4 minutes at room temperature, followed by centrifugation at $300 \times g$ for 5 minutes and 
resuspension of pellets in fresh Essential 8 medium. The cells were then plated at a 1:10 split ratio per passage, on vitronectin coated dishes.

\section{iPSC differentiation and cerebral organoid generation}

Xeno-free iPSCs were differentiated to generate cerebral organoids using the protocol described by Lancaster et al. [18]. Briefly, xeno-free iPSCs were dissociated into singularized cells by incubation in Accutase at room temperature for 4 minutes. Single cell suspensions were plated at 9000 cells/ well into Costar Ultra-Low attachment plates (Corning, USA) in DMEM: F12, 1:100 GlutaMAX, 20\% KOSR, 1:100 NEAA, 1:100 Penicillin/Streptomycin, $0.14 \mathrm{mM} \beta$-mercaptoethanol, $4 \mathrm{ng} / \mathrm{mL}$ b-FGF2 and 50 $\mu \mathrm{M}$ Y-27632 to form embryoid bodies. For organoid generation, embryoid bodies were first primed to commit to the neuroectodermal lineage by transferring into Costar Ultra-Low attachment 24 well plates (Corning, USA) containing neural induction medium, consisting of DMEM: F12, 1:100 GlutaMAX, 1:100 NEAA, 1:100 N2 supplement and $1 \mu \mathrm{g} / \mathrm{mL}$ heparin (Sigma-Aldrich, UK). Two days after transfer, one half volume of medium was added to each well. Six days post-neural induction, neurospheres, displaying the presence of radially organized neuroectoderm were embedded into Matrige ${ }^{\circledast}$ (Corning, USA) droplets and cultured for 5 days in 1:1 DMEM: F12/Neurobasal medium, 1:200 N2, 1:4000 Insulin (Sigma-Aldrich, UK), 1:100 GlutaMAX, 1:200 NEAA, 1:100 Penicillin/Streptomycin, $0.14 \mathrm{mM} \beta$-mercaptoethanol and 1:100 B27 minus Vitamin A.

The organoids were then transferred into $125 \mathrm{~mL}$ spinner bioreactor flasks (Corning, USA) for terminal differentiation in $75 \mathrm{~mL}$ of 1:1 DMEM: F12/Neurobasal medium, 1:200 N2, 1:4000 Insulin, 1:100 GlutaMAX, 1:200 NEAA, 1:100 Penicillin/Streptomycin, $0.14 \mathrm{mM} \beta$-mercaptoethanol and 1:100 XB27 plus Vitamin A. CNS organoid medium was replenished weekly by changing the whole $75 \mathrm{~mL}$ volume.

\section{Immunocytochemistry}

iPSCs (from passage 13 onwards) were characterised for the expression of the pluripotency markers 
SOX2 and TRA1-60 using the PSC Immunocytochemistry kit, as described by the manufacturer. Embryoid bodies differentiated on $0.2 \%$ gelatin (Sigma-Adrich, UK) coated plates for 21 days were stained for the presence of germ layer markers SMA (smooth muscle actin, mesoderm), AFP (Alphafetoprotein, endoderm) and Tuj1 ( $\beta$-III tubulin, ectoderm) using the 3-Germ Layer Immunocytochemistry kit, according to the manufacturer's recommendations. Cerebral organoids harvested at pre-defined timepoints (63 and 92 days) were prepared for immunohistochemistry as described by Lancaster et al. [18]. Briefly, organoids were pre-fixed in $4 \%$ paraformaldehyde (SantaCruz Biotechnologies, USA) at room temperature for 10 minutes and embedded in a $7.5 \%(\mathrm{w} / \mathrm{v})$ gelatin/ $12 \%(\mathrm{w} / \mathrm{v})$ sucrose solution (Sigma-Aldrich, UK) which was allowed to polymerize at $4^{\circ} \mathrm{C}$ for 20 minutes. Embedded organoids were snap frozen in Neg-50 Frozen Section Medium at $-60^{\circ} \mathrm{C}$ for two minutes using a dry ice and isopentane (Sigma-Aldrich, UK) bath and sectioned at $20 \mu \mathrm{m}$ using a Leica CM190 cryostat (Leica, Germany). Sections were mounted on Superfrost ${ }^{\mathrm{TM}}$ Plus Adhesion slides, fixed with $4 \%$ paraformaldehyde for 15 minutes and permeabilized with $1 \%(w / v)$ Saponin (Sigma-Aldrich, UK) for 15 minutes, all at room temperature. A 3\% (w/v) bovine serum albumin (Sigma-Aldrich, UK) blocking solution was then added for 30 minutes at room temperature. Primary antibodies for neural markers were diluted in blocking solution, added to the cells and incubated for 24 hours at $4^{\circ} \mathrm{C}$. The slides were washed 3 times for 10 minutes in phosphate buffered saline (PBS) and then incubated for 1-hour at room temperature with fluorescently conjugated secondary antibodies diluted in blocking solution. The slides were washed 3 times with PBS for 10 minutes, followed by the addition of $2 \mu \mathrm{g} / \mathrm{mL}$ Hoechst 33342 (Sigma-Aldrich, UK) for 10 minutes. Primary antibodies with the following dilutions were used: SOX2 (rat/1:100), Tuj1 (mouse IgG2/1:100, R\&D Bioscience, UK), Nestin (mouse IgG1/1:100, R\&D Bioscience, UK), GFAP mouse IgG1/1:100, R\&D Bioscience, UK), O4 (mouse IgM/1:100, R\&D Bioscience, UK) and MBP (mouse IgG2b/1:100, Abcam, UK). Secondary antibodies and dilutions were: donkey-anti rat (488 AlexaFluor/ 1:250), goat-anti mouse IgG (555 AlexaFluor/ 1:200, Abcam, UK) and goat- anti mouse IgM (550 DyLight/1:200, Abcam, UK). 


\section{Luxol Fast Blue myelin staining}

The chromogenic Luxol Fast Blue staining kit (Abcam, UK) was used to confirm the detection of myelin fibres in organoid cryosections at day 63 and 92, obtained as described above. Organoid sections were immersed in Luxol Blue for 24 hours at room temperature. Tissues were then rinsed by dipping in distilled water, and sections differentiated by dipping several times for a total of 20 seconds in lithium carbonate. Differentiation was continued by repeatedly dipping the slides in $70 \%$ (v/v) ethanol, followed by rinsing with distilled water and immersion in Cresyl Echt violet for 5 minutes at room temperature. The tissues were then rinsed in distilled water and dehydrated in three quick changes of absolute ethanol. Stained tissues were mounted in Histomount ${ }^{\mathrm{TM}}$ medium (Agar Scientific, UK) for imaging.

\section{Imaging}

Stained IPSCs and differentiated embryoid bodies were imaged by wide-field epifluorescence microscopy using a Nikon TS-100 (Nikon, Japan). Immunolabelled organoid sections were imaged using a laser confocal scanning microscope Nikon A1R (Nikon, Japan). Luxol Fast blue stained sections were imaged using a Nikon TS-100 under bright field microscopy.

\section{Western blot}

Thymidine phosphorylase expression was determined by immunoblotting. Protein lysates in Radioimmunoprecipitation assay buffer (Sigma-Aldrich, UK) were diluted 1:4 with Laemmli (BioRad, UK) and heat denatured at $95^{\circ} \mathrm{C}$ for 5 minutes. Twenty-microliters (equating to $5 \mu \mathrm{g}$ protein) of denatured samples and $10 \mu \mathrm{L}$ PAGE ruler marker were loaded onto pre-cast NuPAGE Bis-Tris 4-12\% SDS polyacrylamide $1 \mathrm{~mm}$ gels and run at $80 \mathrm{~mA}$ for 1 hour in $1 \mathrm{x}$ NUPAGE MOPS running buffer. Samples were transferred onto a $0.45 \mu \mathrm{m}$ polyvinylidene difluoride (Bio-Rad, UK) membrane for 45 minutes at $350 \mathrm{~mA}$ in 1x Transfer buffer (25mM Tris, $192 \mathrm{mM}$ glycine and 10\% methanol, pH 8.3, 
Sigma Aldrich, UK). Membranes were blocked with $5 \%$ milk in Tris buffered saline and Tween-20 (Sigma-Aldrich, UK) for 30 minutes and then incubated overnight at $4{ }^{\circ} \mathrm{C}$ with mouse monoclonal anti-thymidine phosphorylase antibodies (1:500, Abcam, UK) or with primary rabbit anti-human pan actin (1:1000, Sigma-Aldrich, UK). Membranes were then incubated with goat anti-mouse $(1: 10,000$, Abcam, UK) and donkey anti-rabbit (1: 10,000, Jackson Laboratories, USA) conjugated with horseradish peroxidase for 1 hour at room temperature. Membranes were incubated with Immobilon Western (Millipore, USA) and CL-Xposure X-ray films, which were exposed for 20 seconds in a dark room.

\section{Episomal plasmid detection}

To detect the presence of the episomal reprogramming plasmids in iPSCs, total DNA from xeno-free cells was extracted using the PureLink Genomic DNA mini kit according to the manufacturer's protocol. Twenty nanograms of DNA were amplified by polymerase chain reaction (PCR) using primers complimentary to the EBNA1 gene cloned in the backbone of the reprogramming plasmids. Sequences for the EBNA1 primers were taken from Okita et al. [19]. Endpoint PCR was performed using the Fast III Brilliant SYBR master mix (Agilent, USA) and the Aria Mx thermocycler (Agilent, USA) as recommended by the manufacturer. Amplified PCR products were loaded onto E-Gel precast $2 \%$ agarose gels with SYBR safe DNA stain and run electrophoretically along with the E-Gel 50 bp DNA ladder for 19 minutes. Gels were imaged using the G:BOX Chemi XX6 (Syngene, UK).

\section{Karyotyping}

Karyotyping was performed on generated iPSCs to ascertain that reprogramming did not result in the acquisition of chromosomal aberrations. Cells cultured on vitronectin coated glass coverslips were harvested at $70 \%$ confluency and incubated with a bromodeoxyuridine $(6 \mathrm{mg} / \mathrm{mL}, \mathrm{Sigma-}$ Aldrich, UK)/colcemid $(0.6 \mu \mathrm{g} / \mathrm{mL}$, Sigma-Aldrich, UK) solution overnight. Cells were then lysed by incubation in 1\% w/v tri-sodium citrate (Sigma-Aldrich, UK) for 20 minutes, and subsequently fixed in $75 \%$ methanol/ $25 \%$ acetic acid (v/v, Sigma-Aldrich, UK), twice, each for 3 minutes. G-banding was 
performed by exposing metaphases in a $0.02 \%$ trypsin solution for 2 minutes and subsequently stained with Giemsa (Sigma-Aldrich, UK) for 10 minutes. Metaphases were visualised under a Leica DM500 LED microscope (Leica, Germany) and chromosomes were sorted using Cytovision v7.2 software. 


\section{Results}

\section{iPSC characterisation}

Immunohistochemical staining of pluripotency markers SOX2 and TRA-1-60 indicated that reprogrammed cells were bona fide pluripotent cells (Figure $1 \mathrm{~A}$ and B). iPSCs also demonstrated the plasticity to differentiate into endoderm, mesoderm and ectoderm tissues as evidence by positive staining for AFP, SMA and Tuj1, respectively, in both MNGIE and control iPSCs (Figure 1 C and D). Karyotyping did not reveal chromosomal abnormalities, with all cells showing 23 pairs of chromosomes (Figure $1 \mathrm{E}$ and F). In early passage iPSCs (< passage 9), amplicons for EBNA1 can be observed in agarose gels, however in late passages (> passage 13) EBNA1 sequences were no longer detectable (Figure $1 \mathrm{G}$ ) indicating loss of episomal vectors.

Immunohistochemical analysis of neural markers

Immunohistochemical staining confirmed the presence of the main neural and glial cell types, namely neural stem cells, neurons, oligodendrocyte and astrocyte precursors as evidenced by positive fluorescence signals for the markers SOX2 and Nestin, Tuj1, O4 and GFAP respectively (Figure 2). The cells displayed the expression of the neural markers co-localizing with the background chromatin (DNA) staining. The organoids displayed the presence of myelin as shown by positive staining for MBP (myelin basic protein), Figure 2.

\section{Luxol Fast Blue myelin staining}

The presence of myelinated fibres in the both MNGIE and control organoid sections can be observed as evidenced by the positive staining for white matter in blue, contrasted by the purple staining of neurons and Nissl bodies, Figure 3. 


\section{Thymidine phosphorylase expression}

Western blot analysis showed the presence of the protein in healthy control PBMCs and Day 92 organoids, although no expression was observed in healthy control iPSCs, Figure 4. Consistent with the clinical phenotype, thymidine phosphorylase was absent in MNGIE PBMCs, iPSCs and Day 92 organoids (Figure 4). Thymidine phosphorylase was also absent in the skeletal muscle negative control. The endogenous control pan actin was expressed in all cell types, Figure 4. 


\section{Discussion}

This is a proof of concept study to determine whether cerebral organoids could be successfully generated and characterised from MNGIE and healthy control derived-iPSCs, and whether the organoids qualified as a bona fide model for MNGIE. The latter was of particular interest due to the ambiguity of reports cited in the literature with regard to their potential for recapitulating late brain development milestones, for example the presence of differentiate glial cells and myelination [2026].

A MNGIE cell line was developed using patient-derived iPSC technology presenting a novel in vitro model of MNGIE. The validation experiments confirmed that the iPSC lines were bona fide pluripotent cells as evidenced by the expression of pluripotency markers and by their plasticity to differentiate in the germ layer tissues. Furthermore, iPSCs displayed epigenetic reprogramming, retaining their pluripotency after the gradual loss of the episomal reprogramming vectors, which occurred through cell division within 13 passages. To fully characterise this novel MNGIE model, an evaluation of mtDNA copy number and mutations in generated cell lines would be required.

Characterised iPSCs were then successfully used for the generation of MNGIE cerebral organoids. Based on qualitative observations, it was possible to conclude that the organoids displayed the presence of cells relevant to the CNS, and most importantly, it was possible to show that cerebral organoids exhibit the expression of astrocytes and myelinating oligodendrocytes, as evidenced by the immunofluorescence detection of GFAP, $\mathrm{O} 4$ and MBP. Furthermore, since MBP isoforms such as MBP-1 and Golli-MBP are known to be expressed in early embryogenesis prior to the beginning of myelination, Luxol Fast Blue staining complemented the immunofluorescence staining of MBP, thus confirming the presence of lipid rich myelin fibres surrounding neurons [27]. 
The expression of thymidine phosphorylase was consistent with the phenotypes of the cell lines, whereby MNGIE cells did not express the enzyme. Healthy control PBMCs and Day 92 organoids showed expression of thymidine phosphorylase, although the protein was not expressed in control iPSCs; we hypothesised that since iPSCs are highly proliferative cells, they would rely on the de novo nucleotide synthesis, hence downregulating the expression of thymidine phosphorylase [28]. In addition to demonstrating the absent expression of thymidine phosphorylase protein in MNGIE cells, we are investigating the presence of enzyme activity in cell lysates.

In the CNS of MNGIE patients, leukoencephalopathy is invariably observed, presenting as a mild decrease in periventricular white matter as shown by MRI $[1,29]$. The model is currently being utilised to examine the alteration in neural cell populations within the organoids and establish if there is a difference in myelin expression with regard to the white matter involvement associated with MNGIE. From the observation of our immunohistochemical data and Luxol Fast Blue staining it was not possible to detect a different pattern of myelination in MNGIE organoids compared to healthy control, reinforcing the hypothesis that in MNGIE, white matter lesions may not be the result of intrinsic molecular derangements but could rather stem from an increased permeability of the blood brain barrier (BBB), resulting in vasogenic oedema and appearing as leukoencephalopathy on MRI $[29,30]$. To examine this, our organoid model is being exploited for the generation novel hypotheses in relation to the possible BBB involvement in MNGIE through the investigation of transcriptomic profiling of MNGIE neural cells for the identification of the pathomolecular mechanisms in the CNS.

In conclusion, cerebral organoids developed in this study, displayed the presence of features of interest for investigating MNGIE, including the expression of astrocytes, oligodendrocytes and myelin markers. Cerebral organoids can therefore be considered as a potentially viable in vitro model for studying the CNS involvement in patients with MNGIE. 
Bibliography

1. Nishino I, Spinazzola A, Papadimitriou A, Hammans S, Steiner I, Hahn CD, Connolly AM, Verloes A, Guimaraes J, Maillard I, et al: Mitochondrial neurogastrointestinal encephalomyopathy: an autosomal recessive disorder due to thymidine phosphorylase mutations. Ann Neurol 2000, 47:792-800.

2. Hirano M, Nishigaki Y, Marti R: Mitochondrial neurogastrointestinal encephalomyopathy (MNGIE): a disease of two genomes. Neurologist 2004, 10:8-17.

3. Hirano M, Silvestri G, Blake DM, Lombes A, Minetti C, Bonilla E, Hays AP, Lovelace RE, Butler I, Bertorini TE, et al.: Mitochondrial neurogastrointestinal encephalomyopathy (MNGIE): clinical, biochemical, and genetic features of an autosomal recessive mitochondrial disorder. Neurology 1994, 44:721-727.

4. Marti R, Nishigaki $\mathrm{Y}$, Hirano $\mathrm{M}$ : Elevated plasma deoxyuridine in patients with thymidine phosphorylase deficiency. Biochem Biophys Res Commun 2003, 303:14-18.

5. Nishigaki Y, Martí R, Copeland WC, Hirano M: Site-specific somatic mitochondrial DNA point mutations in patients with thymidine phosphorylase deficiency. Journal of Clinical Investigation 2003, 111:1913-1921.

6. Nishigaki Y, Marti R, Hirano M: ND5 is a hot-spot for multiple atypical mitochondrial DNA deletions in mitochondrial neurogastrointestinal encephalomyopathy. Hum Mol Genet 2004, 13:91-101.

7. Pontarin G, Gallinaro L, Ferraro P, Reichard P, Bianchi V: Origins of mitochondrial thymidine triphosphate: Dynamic relations to cytosolic pools. Proceedings of the National Academy of Sciences 2003, 100:12159-12164.

8. Rampazzo C, Gallinaro L, Milanesi E, Frigimelica E, Reichard P, Bianchi V: A deoxyribonucleotidase in mitochondria: Involvement in regulation of dNTP pools and possible link to genetic disease. Proceedings of the National Academy of Sciences of the United States of America 2000, 97:8239-8244.

9. Rampazzo C, Ferraro P, Pontarin G, Fabris S, Reichard P, Bianchi V: Mitochondrial deoxyribonucleotides, pool sizes, synthesis, and regulation. J Biol Chem 2004, 279:1701917026.

10. Haraguchi $M$, Tsujimoto $H$, Fukushima M, Higuchi I, Kuribayashi $H$, Utsumi $H$, Nakayama A, Hashizume $\mathrm{Y}$, Hirato J, Yoshida $\mathrm{H}$, et al: Targeted deletion of both thymidine phosphorylase and uridine phosphorylase and consequent disorders in mice. Mol Cell Biol 2002, 22:52125221.

11. Lopez LC, Akman HO, Garcia-Cazorla A, Dorado B, Marti R, Nishino I, Tadesse S, Pizzorno G, Shungu $D$, Bonilla $E$, et al: Unbalanced deoxynucleotide pools cause mitochondrial DNA instability in thymidine phosphorylase-deficient mice. Hum Mol Genet 2009, 18:714-722.

12. Garcia-Diaz B, Garone C, Barca E, Mojahed H, Gutierrez P, Pizzorno G, Tanji K, Arias-Mendoza F, Quinzii CM, Hirano M: Deoxynucleoside stress exacerbates the phenotype of a mouse model of mitochondrial neurogastrointestinal encephalopathy. Brain 2014, 137:1337-1349.

13. Avior Y, Sagi I, Benvenisty N: Pluripotent stem cells in disease modelling and drug discovery. Nat Rev Mol Cell Biol 2016, 17:170-182. 
14. Dutta D, Heo I, Clevers H: Disease Modeling in Stem Cell-Derived 3D Organoid Systems. Trends in Molecular Medicine 2017, 23:393-410.

15. Filosto M, Scarpelli M, Tonin P, Testi S, Cotelli MS, Rossi M, Salvi A, Grottolo A, Vielmi V, Todeschini $A$, et al: Pitfalls in diagnosing mitochondrial neurogastrointestinal encephalomyopathy. J Inherit Metab Dis 2011, 34:1199-1203.

16. Bax $B E$, Bain $M D$, Scarpelli $M$, Filosto $M$, Tonin $P$, Moran $N$ : Clinical and biochemical improvements in a patient with MNGIE following enzyme replacement. Neurology 2013, 81:1269-1271.

17. Okita K, Yamakawa T, Matsumura Y, Sato Y, Amano N, Watanabe A, Goshima N, Yamanaka S: An Efficient Nonviral Method to Generate Integration-Free Human-Induced Pluripotent Stem Cells from Cord Blood and Peripheral Blood Cells. STEM CELLS 2013, 31:458-466.

18. Lancaster MA, Knoblich JA: Generation of cerebral organoids from human pluripotent stem cells. Nat Protocols 2014, 9:2329-2340.

19. Okita K, Matsumura $Y$, Sato $Y$, Okada A, Morizane A, Okamoto S, Hong H, Nakagawa $M$, Tanabe $\mathrm{K}$, Tezuka $\mathrm{K}-\mathrm{i}$, et al: A more efficient method to generate integration-free human iPS cells. Nat Meth 2011, 8:409-412.

20. Kelava I, Lancaster Madeline A: Stem Cell Models of Human Brain Development. Cell Stem Cell, 18:736-748.

21. Mariani J, Simonini MV, Palejev D, Tomasini L, Coppola G, Szekely AM, Horvath TL, Vaccarino FM: Modeling human cortical development in vitro using induced pluripotent stem cells. Proceedings of the National Academy of Sciences of the United States of America 2012, 109:12770-12775.

22. Pasca AM, Sloan SA, Clarke LE, Tian Y, Makinson CD, Huber N, Kim CH, Park J-Y, O'Rourke NA, Nguyen KD, et al: Functional cortical neurons and astrocytes from human pluripotent stem cells in 3D culture. Nat Meth 2015, 12:671-678.

23. Camp JG, Badsha F, Florio M, Kanton S, Gerber T, Wilsch-Bräuninger M, Lewitus E, Sykes A, Hevers $W$, Lancaster $M$, et al: Human cerebral organoids recapitulate gene expression programs of fetal neocortex development. Proceedings of the National Academy of Sciences 2015, 112:15672-15677.

24. Qian X, Nguyen Ha N, Song Mingxi M, Hadiono C, Ogden Sarah C, Hammack C, Yao B, Hamersky Gregory R, Jacob F, Zhong C, et al: Brain-Region-Specific Organoids Using Minibioreactors for Modeling ZIKV Exposure. Cell 2016, 165:1238-1254.

25. Monzel AS, Smits LM, Hemmer K, Hachi S, Moreno EL, van Wuellen T, Jarazo J, Walter J, Brüggemann I, Boussaad I, et al: Derivation of Human Midbrain-Specific Organoids from Neuroepithelial Stem Cells. Stem Cell Reports 2017, 8:1144-1154.

26. Matsui TK, Matsubayashi M, Sakaguchi YM, Hayashi RK, Zheng C, Sugie K, Hasegawa M, Nakagawa T, Mori E: Six-month cultured cerebral organoids from human ES cells contain matured neural cells. Neuroscience Letters 2018, 670:75-82.

27. Tosic M, Rakic S, Matthieu J, Zecevic N: Identification of Golli and myelin basic proteins in human brain during early development. Glia 2002, 37:219-228.

28. Lane AN, Fan TWM: Regulation of mammalian nucleotide metabolism and biosynthesis. Nucleic Acids Research 2015, 43:2466-2485.

29. Scarpelli M, Ricciardi GK, Beltramello A, Zocca I, Calabria F, Russignan A, Zappini F, Cotelli MS, Padovani A, Tomelleri G, et al: The Role of Brain MRI in Mitochondrial Neurogastrointestinal Encephalomyopathy. The neuroradiology journal 2013, 26:520-530.

30. Szigeti K, Sule N, Adesina AM, Armstrong DL, Saifi GM, Bonilla E, Hirano M, Lupski JR: Increased blood-brain barrier permeability with thymidine phosphorylase deficiency. Annals of Neurology 2004, 56:881-886. 


\section{Figure legend}

Figure 1- Characterisation of MNGIE and control iPSCs. Wide-field fluorescence micrographs of the pluripotency markers SOX2 and TRA-1-60 in A) MNGIE and B) control iPSCs, 10x magnification, scale bar 100 $\mu \mathrm{m}$. Wide-field fluorescence micrographs of germ layer markers Tuj1 (ectoderm), AFP (endoderm) and SMA (mesoderm) in C) MNGIE and D) control iPSCs, 40x magnification, scale bar $50 \mu \mathrm{m}$. G-banding in E) MNGIE and F) control iPSCs displaying a normal karyotype and XY sex chromosomes. G) Agarose gel electrophoresis of PCR amplicons of EBNA1 sequences (61 bp) specific to the episomal plasmid backbone.

Figure 2- Immunofluorescence staining for neural cells. Sections of A) MNGIE and B) Control organoids at day 63 and 92 of development were stained for the neural stem cell markers SOX2 (green) and Nestin (red), the astrocyte marker GFAP (red), the neuronal marker Tuj1 (red), the oligodendrocyte marker $\mathrm{O} 4$ (red) and the myelin marker MBP (red). Neural markers co-localized with the chromatin stain (blue). Magnification 20x, Scale bar $100 \mu \mathrm{m}$.

Figure 3- Luxol Fast Blue myelin staining in cerebral organoids. Sections of MNGIE and Control organoids at day 63 and 92 of development were stained using Luxol Fast Blue to stain lipid rich myelin fibres (blue) and Cresyl Echt violet to stain neurons (purple). Presence of myelin fibres (arrows) was observed in both MNGIE and Control organoids. Magnification 40x, Scale bar $50 \mu \mathrm{m}$.

Figure 4- Thymidine phosphorylase protein expression. Western blot analysis shows the expression of the 50 kDa thymidine phosphorylase homodimer in cell lysates of healthy control A) PBMC and B) Day 92 cerebral organoids but not in control iPSCS (A). MNGIE cells did not display thymidine phosphorylase in PBMCs, iPSCs (A) or Day 92 organoids (B). Human skeletal muscle satellite cells (HsKMSC) negative control did not show positive bands for the protein. The $45 \mathrm{kDa}$ pan actin was probed as a loading control displaying positive bands in all samples. 
A
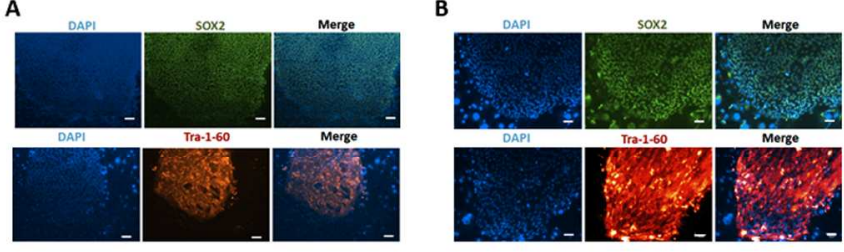

C DAPI
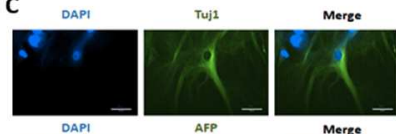

Merge
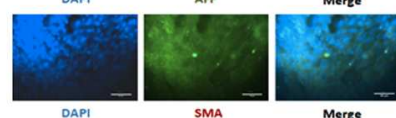

Merge
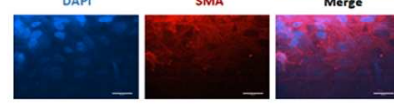

E

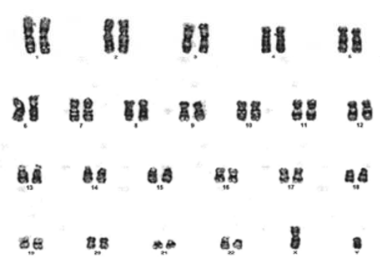

D DAPI Tuj 1

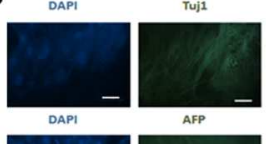

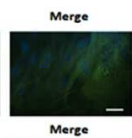
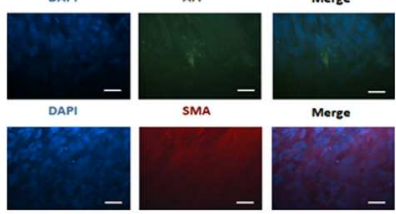

$\mathbf{F}$

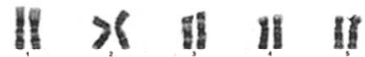

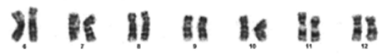

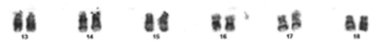

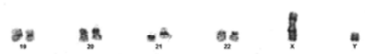

G

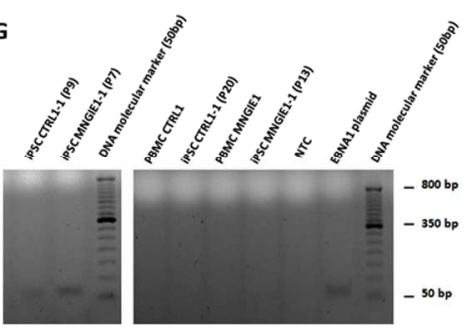

Figure 1- Characterisation of MNGIE and control iPSCs. Wide-field fluorescence micrographs of the pluripotency markers SOX2 and TRA-1-60 in A) MNGIE and B) control iPSCs, 10x magnification, scale bar $100 \mu \mathrm{m}$. Wide-field fluorescence micrographs of germ layer markers Tuj1 (ectoderm), AFP (endoderm) and SMA (mesoderm) in C) MNGIE and D) control iPSCs, 40x magnification, scale bar $50 \mu \mathrm{m}$. G-banding in E) MNGIE and F) control iPSCs displaying a normal karyotype and XY sex chromosomes. G) Agarose gel electrophoresis of PCR amplicons of EBNA1 sequences (61 bp) specific to the episomal plasmid backbone.

$$
190 \times 338 \mathrm{~mm}(300 \times 300 \mathrm{DPI})
$$




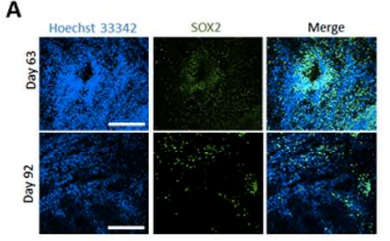

C

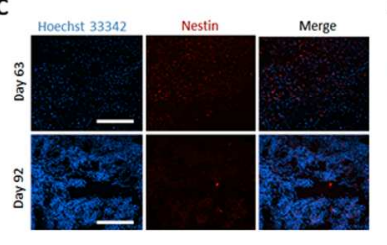

E

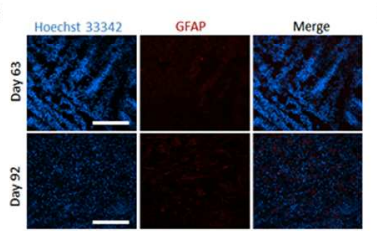

G
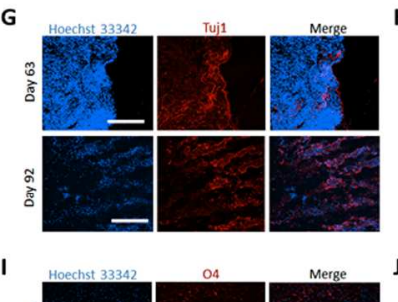

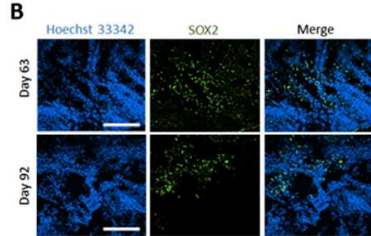

D
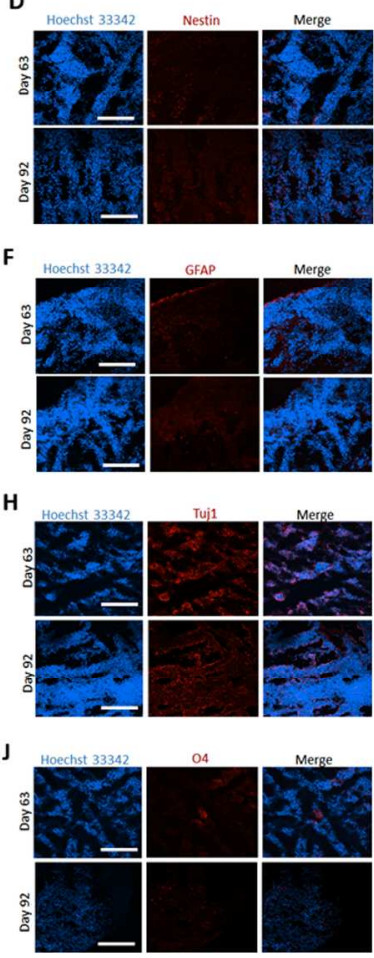

K
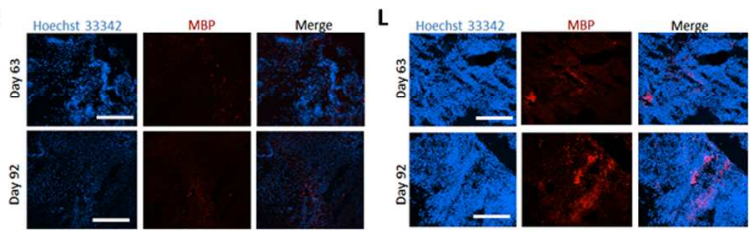

Figure 2- Immunofluorescence staining for neural cells. Sections of A) MNGIE and B) Control organoids at day 63 and 92 of development were stained for the neural stem cell markers SOX2 (green) and Nestin (red), the astrocyte marker GFAP (red), the neuronal marker Tuj1 (red), the oligodendrocyte marker O4 (red) and the myelin marker MBP (red). Neural markers co-localized with the chromatin stain (blue). Magnification 20x, Scale bar $100 \mu \mathrm{m}$.

$190 \times 338 \mathrm{~mm}(300 \times 300$ DPI $)$ 
Figure 3- Luxol Fast Blue myelin staining in cerebral organoids. Sections of MNGIE and Control organoids at day 63 and 92 of development stained with Luxol Fast Blue and Cresyl Echt violet showed lipid rich myelin fibres (blue) and neurons (purple). Presence of myelin fibres (arrows) was observed in both MNGIE and Control organoids. Magnification 40x, Scale bar $50 \mu \mathrm{m}$.

$164 \times 132 \mathrm{~mm}(300 \times 300$ DPI $)$

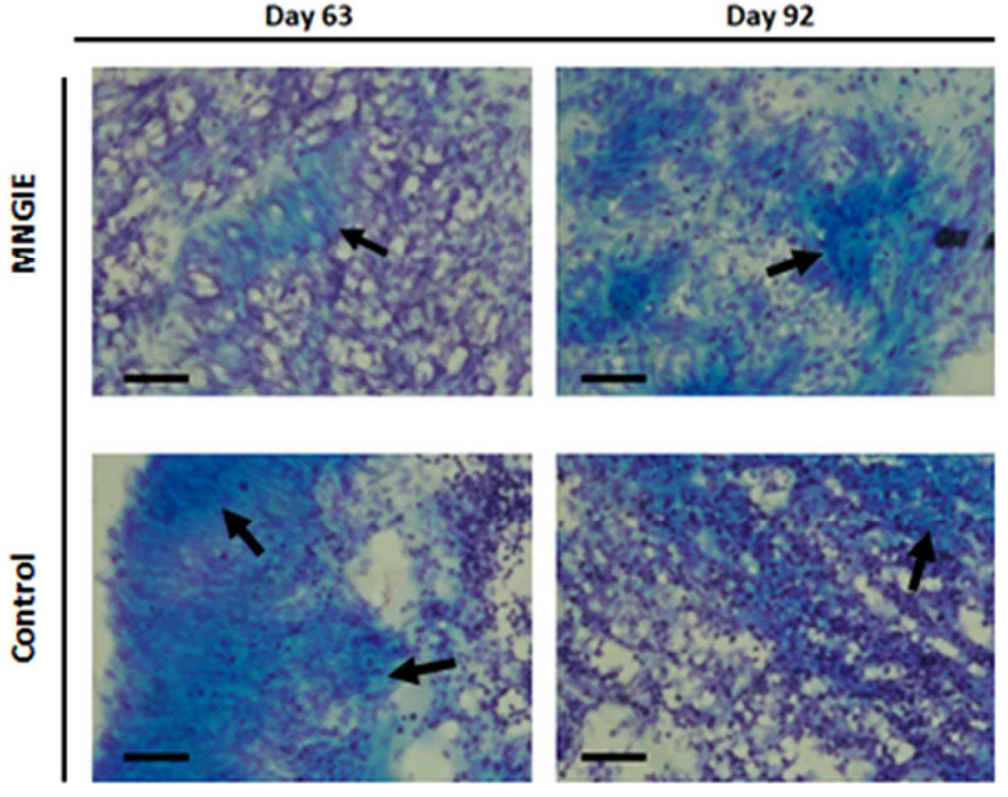


A

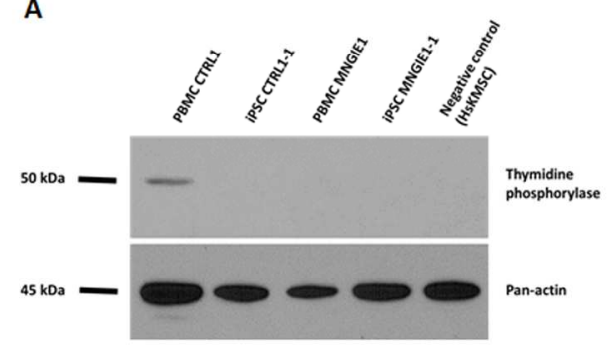

B

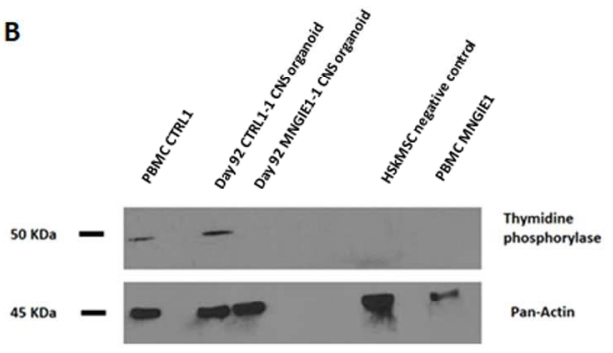

Figure 4- Thymidine phosphorylase protein expression. Western blot analysis shows the expression of the 50 kDa thymidine phosphorylase homodimer in cell lysates of healthy control A) PBMC and B) Day 92 cerebral organoids but not in control iPSCs (A). MNGIE cells did not display thymidine phosphorylase in PBMCs, iPSCs (A) or Day 92 organoids (B). Human skeletal muscle satellite cells (HsKMSC) negative control did not show positive bands for the protein. The $45 \mathrm{kDa}$ pan actin was probed as a loading control displaying positive bands in all samples.

$237 \times 81 \mathrm{~mm}(300 \times 300$ DPI $)$ 